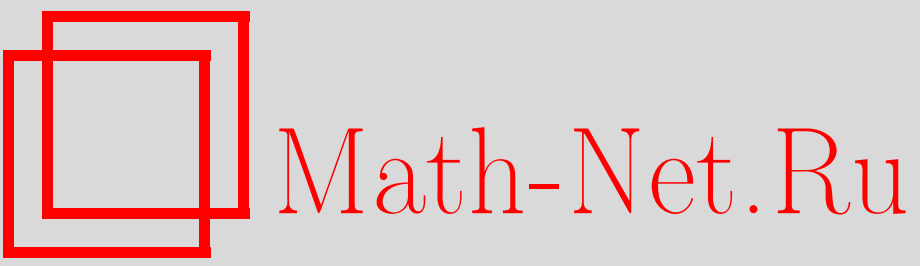

С. В. Савченко, О спектрах конечных подматриц бесконечных неразложимых матриц, УМН, 1997, том 52, выпуск 3, 175-176

DOI: https://doi.org/10.4213/rm858

Использование Общероссийского математического портала Math-Net.Ru подразумевает, что вы прочитали и согласны с пользовательским соглашением

http://www.mathnet.ru/rus/agreement

Параметры загрузки:

IP: 3.80 .253 .173

26 апреля 2023 г., 18:04:59 


\title{
О СПЕКТРАХ КОНЕЧНЫХ ПОДМАТРИЦ БЕСКОНЕЧНЫХ НЕРАЗЛОЖИМЫХ МАТРИЦ
}

\author{
C. В. САВЧЕНКО
}

Пусть $A=\left(a_{i j}\right)$ - конечная или бесконечная матрица с комплексньми элементами $a_{i j}$. Каждой такой матрице $A$ сопоставим ориентированный граф̆ $G(A)$, множество вершин которого совпадает с множеством индексов элементов матрицы, а множество ребер определяется по следующему правилу: из $i$ идет ребро в $j$ тогда и только тогда, когда $a_{i j} \neq 0$. Если графф $G(A)$ связен, то матрица $A$ называется неразложимой.

Для любой конечной последовательности индексов $\gamma=\left\{i_{k}\right\}_{k=0}^{n}$ длины $n$ положим $Q_{A}(\gamma)=$ $a_{i_{0} i_{1}} \cdots a_{i_{n-1} i_{n}}$. Обозначим через $a(A, i, n)$ сумму величин $Q_{A}(\gamma)$ по всем $\gamma$ длины $n$, у которых $i_{0}=i_{n}=i, i_{k} \neq i$ при $k=1, \ldots, n-1$. Положим

$$
\varphi(A, i, z)=\sum_{n=1}^{\infty} a(A, i, n) z^{n} .
$$

Пусть $r(A, i)$ - радиус сходимости этого ряда. В дальнейшем мы будем рассматривать класс $\mathscr{F}$ неразложимых матриц $A$ с неотрицательными элементами, удовлетворяющих условию $r(A, i)>0$ при некотором (а следовательно, при любом) $i$. Пусть $R(A, i)$ - единственное решение уравнения $\varphi(A, i, z)=1$ на интервале $(0, r(A, i))$, если $\varphi(A, i, r(A, i))>1$, и $R(A, i)=r(A, i)$ в противном случае. Для неразложимой матрицы $A$ величина $R(A, i)$ не зависит от $i$. Поэтому мы будем обозначать ее просто $R(A)$. Для конечной матрицы $A$ положим $\lambda(A)=\max \{|\lambda|: \lambda \in \operatorname{Spec}(A)\}$, где $\operatorname{Spec}(A)-$ спектр $A$. Для бесконечной матрицы $A$ величину $\lambda(A)$ определим как супремум $\lambda\left(A^{\prime}\right)$ по всем ее конечным подматрицам $A^{\prime}$. Величины $\lambda(A)$ и $R(A)$ связаны соотношением $\lambda(A)=R(A)^{-1}[1]$.

Положим $r(A)=\sup _{i} r(A, i)$. Если $r(A)>R(A)$ (это условие эквивалентно неравенству $\varphi(A, i, r(A, i))>1$ при некотором (а следовательно, при любом) $i)$ для $A \in \mathscr{F}$, то множество $V^{\max }(A)$ тех $i$, для которых $r(A, i)=r(A)$, непусто и конечно. Пусть

$$
J(A, i)=\{z: \varphi(A, i, z)=1,|z|<r(A, i)\}
$$

(число копий точки $z$ в множестве $J(A, i)$ совпадает с кратностью $z$ как единицы функции $\varphi(A, i, z))$. Испольуя резулштаты работы [2], нетрудно показать, что $J(A, j)=J(A, i) \cap\{z$ : $|z|<r(A, j)\}$ при любом $j$, если $i \in V^{\max }(A)$. Поэтому имеет смысл положить $J(A)=J(A, i)$, где $i$ - любой элемент множества $V^{\max }(A)$. Очевидно, множество $J(A)$ непусто $(R(A) \in J(A))$ и в силу аналитичности функций $\varphi(A, i, z)$ в круге $|z|<r(A, i)$ не более чем счетно. В случае конечной матрицы $A$ функция $\varphi(A, i, z)$ рациональна при любом $i$. Поэтому $\varphi(A, i, r(A, i))=\infty$ и, следователшно, $r(A)>R(A)$.

ПредЛОЖенИЕ 1. Пусть матрица $A \in \mathscr{F}$ конечна. Тогда

$$
\operatorname{Spec}(A) \cap\left\{z:|z|>r(A)^{-1}\right\}=J(A)^{-1},
$$

где $J(A)^{-1}=\left\{z^{-1}: z \in J(A)\right\}$. Каждому собственному значению $\lambda \in J(A)^{-1}$ соответствует ровно одна жорданова клетка или, другими словами, каждое собственное значение $\lambda \in J(A)^{-1}$ имеет только один собственньй вектор.

СледствиЕ 1. Пусть $A$ - конечная симметрическая матрица из класса $\mathscr{F}$. Тогда

$$
\begin{aligned}
& \operatorname{Spec}(A) \cap\left(r(A)^{-1}, \lambda(A)\right]=\lambda(A), \\
& \operatorname{Spec}(A) \cap\left[-\lambda(A),-r(A)^{-1}\right)=J(A)^{-1} \cap \mathbb{R}^{-},
\end{aligned}
$$

причем последнее множество или пусто, или состоит из одной точки.

Пусть все наддиагональные и поддиагональные элементы матрицы $A$ порядка 5 равны 1 , а все остальные - нулю. Тогда множество $\operatorname{Spec}(A) \cap\left[-\lambda(A),-r(A)^{-1}\right)$ состоит из единственной 
точки $z=-\lambda(A)$. Если все элементы матрицы $A$ равны единице, то, очевидно, это множество пусто. Заметим, что для первой матрицы величина $r(A)^{-1}$, как и $-r(A)^{-1}$, является простым собственным значением. Таким образом, все случаи, описанные в следствии 1 , имеют место, и величину $r(A)$, вообще говоря, нельзя заменить на меньшее число.

Последовательность подматриц $A_{n}$ матрицы $A$ будем называть исчерпьвающей $A$, если для любых $i$ и $j$ существует такое $N(i, j)$, что $(i j)$-элемент матрицы $A-A_{n}$ равен 0 при $n \geqslant N(i, j)$. Пусть $B_{n}$ и $B$ - конечные подмножества комплексной плоскости $\mathbb{C}$. Будем писать $B_{n} \rightarrow B$, если $\operatorname{card} B_{n}=\operatorname{card} B$ при всех достаточно болшших $n$ и для любого достаточно малого $\varepsilon>0$ существует такое $n(\varepsilon)$, что при $n \geqslant n(\varepsilon)$ для каждой точки $z \in B$ найдется ровно одно $z_{n} \in B_{n}$, для которого $\left|z-z_{n}\right|<\varepsilon$.

Теорема 1. Пусть $A$ - бесконечная матрица из класса $\mathscr{F}$ и $r(A)>R(A)$. Тогда существует такое $\varepsilon(A)>0$, что для любой конечной подматрицы $A^{\prime}$ матрицы $A$ справедливо следующее условие:

$$
\operatorname{Spec}\left(A^{\prime}\right) \cap\left\{z:|z|<\lambda\left(A^{\prime}\right)\right\} \subset\{z:|z|<\lambda(A)-\varepsilon(A)\} .
$$

Кроме того, если $A_{n}$ - последовательность конечных подматриц, исчерпьвающих матрицу $A$, то для любого $\lambda>r(A)^{-1}$ при $n \rightarrow \infty$ имеем

$$
\operatorname{Spec}\left(A_{n}\right) \cap\{z:|z|>\lambda\} \rightarrow J^{-1}(A) \cap\{z:|z|>\lambda\} .
$$

Если бесконечная матрица $A$ с неотрицательными элементами симметрична и $\lambda(A)<\infty$, то она порождает ограниченный самосопряженный оператор на пространстве $l_{2}$. В этом случае под $\operatorname{Spec}(A)$ будем подразумевать спектр этого линейного оператора. Очевидно, его спектральньй радиус совпадает с $\lambda(A)$.

Теорема 2. Пусть $A$ - бесконечная симметрическая матрица из класса $\mathscr{F}$. Тогда точка $z=\lambda(A)$ отделима от остальной части спектра, если и только если $r(A)>$ $R(A)$. В этом случае справедливо утверждение следствия 1.

ЗАмечАниЕ 1 . Если при некотором $i$ особенность функции $\varphi(A, i, z)$ в точке $z=r(A, i)$ отлична от полюса (например, $\varphi(A, i, r(A, i))<\infty)$, то точка $z=r(A, i)^{-1}=r(A)^{-1}$ является предельной точкой множества $\operatorname{Spec}(A)$.

Все вышеизложенные резултаты без труда переносятся на случай неразложимой матрицы $A$ с комплексными элементами, если $\varphi(A, i, z)=\varphi(|A|, i, z)$, где $|A|=\left(\left|a_{i j}\right|\right)$, при некотором (а следователшно, и при любом) $i$. В частности, они справедливы для любой матрицы Якоби $A$ с $\lambda(A)<\infty$ (для матриц Якоби это условие равносильно равномерной ограниченности по модулю всех ее элементов), диагоналшные элементы которой неотрицательны.

\section{СПИСОК ЛИТЕРАТУРЫ}

[1] Gurevich B. M. // Z. Wahrscheinlichkeitstheorie verw. Geb. 1984. V. 68. P. 205-242. [2] Vere-Jones D. // Quart. J. Math. Oxford Ser. (2). 1962. V. 13. P. 7-28. 